\title{
A Finite Element Reduced Order Model based on Adaptive Mesh Refinement and Artificial Neural Networks
}

\author{
Joan Baiges $^{*, 1}$, Ramon Codina ${ }^{1,2}$, Inocencio Castañar ${ }^{1}$ and Ernesto Castillo ${ }^{3}$ \\ ${ }^{1}$ Universitat Politècnica de Catalunya, Jordi Girona 1-3, Edifici C1, 08034 Barcelona, Spain \\ ${ }^{2}$ Centre Internacional de Mètodes Numèrics a l'Enginyeria (CIMNE), \\ Edifici C1, Campus Nord UPC C/ Gran Capità S/N 08034 Barcelona, Spain \\ ${ }^{3}$ Universidad de Santiago de Chile, USACH. Departamento de Ingeniería Mecánica, \\ Av. Bernardo O'Higgins 3363, Santiago de Chile, Chile \\ $*$ joan. baiges@upc. edu
}

March 13, 2019

In this work a reduced order model based on adaptive finite element meshes and a correction term obtained by using an artificial neural network (FAN-ROM) is presented. The idea is to run a high-fidelity simulation by using an adaptively refined finite element mesh, and compare the results obtained with those of a coarse mesh finite element model. From this comparison, a correction forcing term can be computed for each training configuration. A model for the correction term is built by using an artificial neural network, and the final reduced order model is obtained by putting together the coarse mesh finite element model, plus the artificial neural network model for the correction forcing term.

The methodology is applied to non-linear solid mechanics problems, transient quasi-incompressible flows, and a fluid-structure interaction problem. The results of the numerical examples show that the FAN-ROM is capable of improving the simulation results obtained in coarse finite element meshes at a reduced computational cost.

\section{Introduction}

Numerical simulation is nowadays being routinely used in design and optimization in engineering and other scientific fields. However, many times these optimization and design processes are limited by the amount of available computational power: in the absence of other tools and analysis, numerical simulation is often insufficient in itself to address complex physics [24]. The amount of data that comes from a nonlinear evolution and coupled problems could be difficult to manage and, obviously, highly expensive to solve, even with the use of good mesh generators, discretization schemes, and solution algorithms [10]. For accurate simulations, typical finite element codes (for instance) may require several thousands of degrees of freedom, which many times results in the need for using costly supercomputing facilities $[1,9,29]$. In order to overcome this problem, the scientific community has been putting a lot of effort in the development of the so called reduced order models (ROM) in recent years, which has been applied to practically all the areas of computational mechanics. ROMs aim at reproducing, at a low computational cost, the features and accuracy of full order models (FOM), with models with only a few degrees of freedom, but which have an accuracy similar to the FOM.

The idea to reduce the dimensions of the problem has been a field of intense research in the last years. Examples of dimension reduction approaches include separated representations in the framework of Proper Generalized Decompositions (PGD) [12, 13], sparse grid techniques [19, 11], reduced basis and model order reduction based on the Proper Orthogonal decomposition method (POD) $[23,21,28,35,4,5]$ or in the Centroidal Voronoi Tessellation method (CVT) $[18,10]$, among others. The advantages and disadvantages of each approach depend on the specific problem.

The approach presented in this work departs from the ideas presented in [6]. In that work, a reduced order subscales model for Proper Orthogonal Decomposition (POD) was presented, where the subscales 
were defined as a linear function of the solution of the reduced-order model. The coefficients of this linear function were obtained by comparing the solution of the full-order model with the solution of the reduced-order model for the same initial conditions. The main idea was to use a high fidelity simulation and compare the result with that of a POD based reduced order model. The difference between both yielded a correction term which could, firstly, be modeled through a least-squares approach, and secondly be added to the ROM system of equations as a correction term. This correction greatly enhanced the performance of the ROM model, allowing to simulate complex flow behavior and to improve the results when using subintegration through hyper-reduction $([30,31])$.

Similar ideas have been exploited in recent works. In [27], a dynamic closure modeling approach has been proposed to stabilize the projection-based reduced order models in turbulent flows, proving to be a promising technique for different flow conditions. The key idea of that method consist in using an eddy viscosity closure approach to stabilize the resulting surrogate model considering the analogy between large eddy simulation and truncated modal projection. In the same line of closure problem, in [37], a Data-Driven Filtered Reduced Order Model (DDF-ROM) method is proposed for the numerical aproximation of CFD problems. In that work, the closure of the filtered ROM problem is enforced in two steps using a quadratic ansatz to model the interaction between the resolved and unresolved modes. In the same line, in [25], the authors propose a Data-Driven Correction ROM method to ensure the closure of the ROM, enforcing that the ROM problem satisfied the same physical laws that the full order problem.

A well-known weakness of the ROM methods is that they can be neither robust with respect to parameter changes nor cost-effective for handling the non-linear dependence of complex dynamical systems [34]. On this regard, machine learning framework has shown to be a promising tool to enhance the robustness of this numerical technique. In [33], the effect of discarded modes is taken into account using a machine learning architecture based on a single hidden layer feed-forward artificial neural network. This

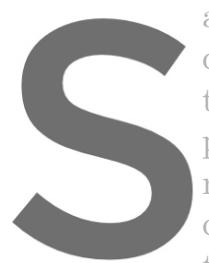
approach allow the auth

coupled flows. In that vo

the capabilities of the pro

physical problem. In the

method for parametrize

collection of high-fidelit

the coefficient of the red
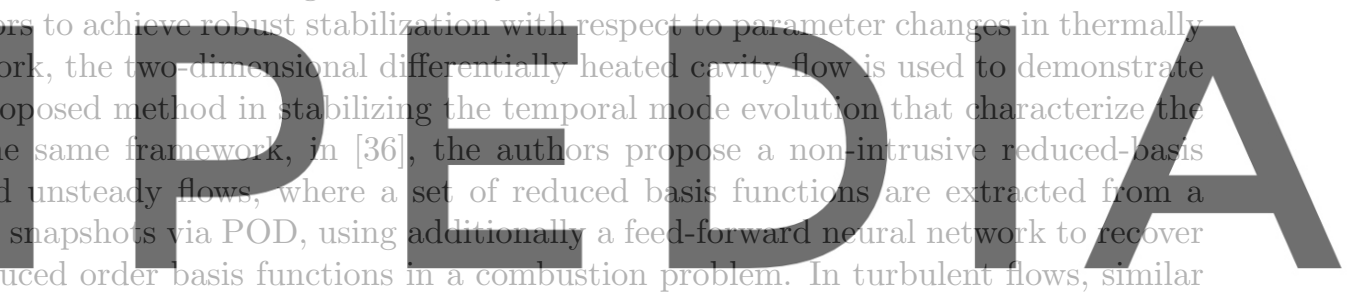

procedures have been proposed based on multi-kernel neural networks [22] and the Extreme Learning

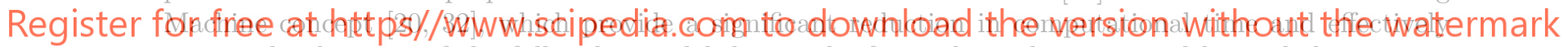
retains the dynamic of the full order model during the forward simulation period beyond the training data set.

In this work, these ideas are extended to the setting of Finite element reduced order models and Adaptive mesh refinement through artificial Neural networks (FAN-ROM): instead of solving a large scale finite element simulation in order to train a POD model, the efforts are going to be focused in two finite element models which depart from a coarse finite element mesh. The first model, the high fidelity model, is going to be adaptively refined using an error estimator. This model will be computationally expensive (although still less costly than a uniformly refined mesh model), and it will be the reference FOM model. The second model is going to be the finite element model in the original coarse mesh. In order to enhance the performance of the low cost model, a correction term is going to be trained by comparing the results of the FOM and ROM models in a set of training configurations, and using an Artificial Neural Network (ANN). This correction term will be added to the ROM finite element equations, which will now yield a result close to the FOM model.

Several advantages with respect to other methodologies can be observed in this approach: firstly, through the usage of hierarchical adaptive mesh refinement, the cost of the high fidelity model is kept as low as possible. These high fidelity simulations can also be run in parallel. Secondly, there is no need to store snapshots for the training of the reduced order model, because the ANN can be continuously trained. Finally, there is no need to build two different approaches for the ROM and the FOM, in both cases a finite element model is used, with a minor modification in the case of the ROM.

The paper is organized as follows: in Section 2 the proposed ROM methodology is presented for a generic problem. Firstly a least-squares approach is described, and secondly the final FAN-ROM method by using artificial neural networks is explained. In Section 3 the methodology is tested in different problems: a non-linear large strain solid mechanics simulation, a nearly incompressible flow problem, and 
a fluid-structure interaction model. Finally some conclusions close the paper in Section 4.

\section{Proposed methodology}

In order to illustrate the proposed method, let us suppose that we want to solve a a simple linear, stationary problem. This would be the case, for instance, of the non-transient heat transfer problem, which yields the Poisson equation. Also, let us suppose that we want to solve it in a given domain, but in a large number of different configurations, which depend on the boundary conditions, the external source terms or the material properties. If an accurate solution is required in each of these configurations, a simple possible approach is to run a simulation for each set of boundary and source term conditions by using a fine mesh finite element model. However, this methodology would be expensive since each of the executions requires of significant computational effort.

The proposed strategy is based on executing the fine mesh simulations only in a small set of configurations, and then extract the appropriate information from these high fidelity solutions in order to train a reduced order model. This reduced order model will be used to accurately approximate the solution in the rest of the configurations. The idea is similar to what is done in Proper Orthogonal Decomposition (POD). However, in the present methodology the reduced order model is simply a coarse finite element model, which will be enhanced by using an artificial neural network.

In order to train the reduced order model, the method consists in building two models of the same physical problem in the so called training configurations. The first model is an adaptively refined finite element mesh, which takes as starting point a coarse mesh. An error estimator is used in order to decide which areas of the finite element mesh need to be recursively refined (see $[3,2,8]$ for the strategy employed by our group). The resulting model is supposed to be very accurate, but also expensive to execute. The second model is the reduced order model, which consists simply in the original coarse finite element model, without any furthe explained. Consequently Let us depart from th each finite element discreti system can be bailt frot in
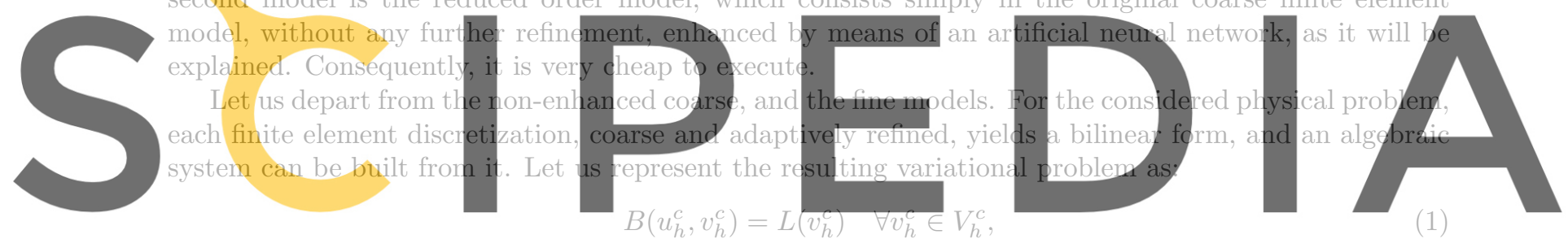

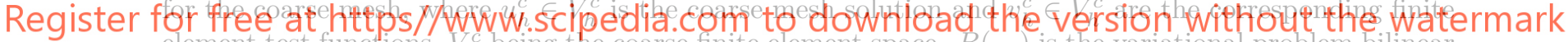
element test functions, $V_{h}^{c}$ being the coarse finite element space. $B(\cdot, \cdot)$ is the variational problem bilinear form, and $L(\cdot)$ its corresponding right hand side. We also denote by $m$ the number of finite element nodes of the coarse mesh. Equivalentily, for the refined mesh, the corresponding problem is:

$$
B\left(u_{h}^{f}, v_{h}^{f}\right)=L\left(v_{h}^{f}\right) \quad \forall v_{h}^{f} \in V_{h}^{f},
$$

where now $u_{h}^{f} \in V_{h}^{f}$ is the fine adaptive mesh solution and $v_{h}^{f} \in V_{h}^{f}$ are the corresponding finite element test functions, $V_{h}^{f}$ being the adaptively refined finite element space. We also denote by $M$ the number of nodes of the fine finite element mesh. It is clear that the solution to both problems is different, and that the fine mesh will yield a more accurate result. The objective now is to be able to modify the coarse mesh system so that an improved solution is obtained.

Let us start by defining a projection operator onto the coarse mesh finite element space $V_{h}^{c}, P_{h}^{c}(\cdot)$. The next step is to define a model enhancement function. For each configuration $i$ (given by the boundary conditions and external source terms), we denote $u_{h}^{c}$ as the solution to (1), and $u_{h}^{f}$ as the solution to (2). Then the model enhancement function is defined as:

$$
\begin{aligned}
C_{i}\left(P_{h}^{c}\left(u_{h}^{f}\right), v_{h}^{c}\right): & =B_{i}\left(P_{h}^{c}\left(u_{h}^{f}\right), v_{h}^{c}\right)-B_{i}\left(u_{h}^{c}, v_{h}^{c}\right) \\
& =B_{i}\left(P_{h}^{c}\left(u_{h}^{f}\right), v_{h}^{c}\right)-L_{i}\left(v_{h}^{c}\right) .
\end{aligned}
$$

Now the solution to:

$$
B_{i}\left(u_{h}, v_{h}^{c}\right)-C_{i}\left(u_{h}, v_{h}^{c}\right)=L_{i}\left(v_{h}^{c}\right) \quad \forall v_{h}^{c} \in V_{h}^{c},
$$

is precisely $u_{h}=P_{h}^{c}\left(u_{h}^{f}\right)$, that is, the projection of the fine mesh solution onto the coarse mesh space. The conclusion is that if we are capable of building a model for $C_{i}\left(u_{h}, v_{h}^{c}\right)$, we will be able to improve the solution of the coarse mesh model and obtain a cheap, accurate reduced order model. 


\subsection{Least Squares approach}

The first approach to build $C_{i}$ is to use a Least Squares method. For a given configuration $i$, the coarse mesh algebraic form of (1) is written as:

$$
A_{i} U_{i}^{c}=F_{i},
$$

where $A_{i}$ is the algebraic matrix form of the bilinear form $B$ for configuration $i$ (determined by a set of boundary conditions), $U_{i}^{c}$ is the coarse, uncorrected vector of unknowns and $F_{i}$ is the algebraic counterpart of $L$ for configuration $i$ (given by a set of external forces). The objective is to add a vector $D_{i}$ such that the following equation holds:

$$
A_{i} U_{i}^{c f}+D_{i}=F_{i},
$$

where $D_{i}$ is the algebraic counterpart of the model function $C_{i}$, evaluated at configuration $i$. $U_{i}^{c f}$ is the algebraic counterpart of $P_{h}^{c}\left(u_{h, i}^{f}\right)$, that is, the coarse mesh projected, fine mesh solution at the given configuration. In the least squares methodology, the model for $D_{i}$ will be linearly dependent with the algebraic solution $U_{i}^{c f}$ :

$$
D_{i} \approx A_{D} U_{i}^{c f}-F_{D}
$$

The corrected reduced order model is now:

$$
\left(A_{i}+A_{D}\right) U=F_{i}+F_{D} .
$$

The question now is how to build $A_{D}$ and $F_{D}$. One of the requirements for matrix $A_{D}$ is that the graph of the matrix must not be denser than the graph of matrices $A_{i}$. Due to this, matrix $A_{D}$ is forced to have the same connectivity pattern as matrices $A_{i}$, that is, the correcting term for a given matrix node will depend only on the values of the unknown in the first layer of surrounding nodes of the coarse finite element mesh, that is, nodes which share an element. Let us suppose that there exist $N$ training

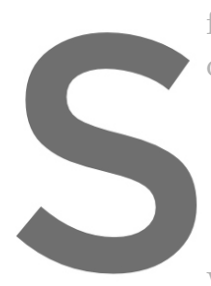

configurations. Then, $\mathrm{m}$

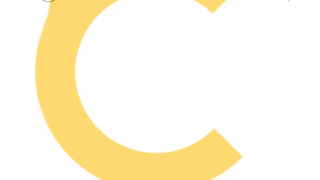

which yields a classical teast squares problem. Note
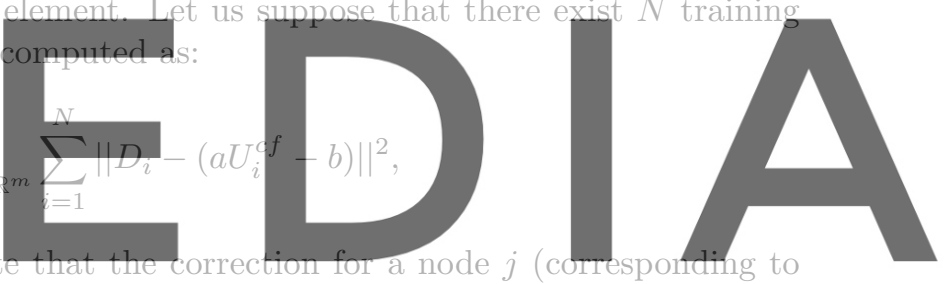

the coefficients in row $j$ of $A_{D}$ and the $j$-th coefficient of $F_{D}$ ) can be computed independently from the

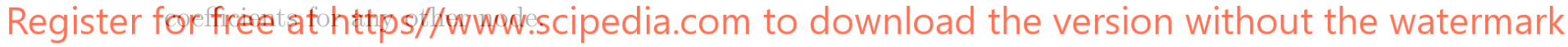

\subsection{Artificial Neural Network approach}

The least squares approach is convenient for linear, stationary problems where the error of a given system does not propagate in successive simulations. However, when applied to non-linear transient problems it can lead to an unstable behavior of the reduced order model because there is no upper or lower limit to the correction it can introduce. A similar behavior was also observed in [7], where a model filtering was required to stabilize the least squares correction model. This is the reason why an artificial neural network to approximate correction vectors $D_{i}$ is proposed. Despite artificial neural network models are able to represent much more complex behaviors than a simple linear least squares approach, they present the inconvenience of being non-linear. This means that it is not possible to build an implicit model such as (3), and this issue makes the approach less suitable for linear stationary problems. On the contrary, in non-linear or transient problems (where each time step can be associated to a configuration), an explicit model based on an artificial neural network can be built. In this case, the correction vector for a given configuration is approximated as:

$$
D_{i} \approx F_{D}\left(U_{i}^{c f}\right)
$$

where $F_{D}\left(U_{i}^{c f}\right)$ represents a trained neural network model which takes as an input $U_{i}^{c f}$ and as an output the $D_{i}$ vector approximation.

Computational effort is one major concern for this model, and this is the reason why building a very complex neural network with $\mathcal{O}(m)$ inputs and $\mathcal{O}(m)$ outputs is avoided, which would have a training and evaluation cost of $\mathcal{O}\left(\mathrm{m}^{2}\right)$. Instead, $\mathcal{O}(\mathrm{m})$ neural networks are trained, one associated to each degree of freedom of the finite element coarse mesh, and each of these neural networks takes as input the values of the unknown in the degrees of freedom in the first layer of surrounding nodes of the coarse finite element 
mesh. The cost of training the artificial neural network is of $\mathcal{O}\left(M \times n_{\text {neigh }}\right)$, where $n_{\text {neigh }}$ is the mean number of first layer neighbors for each node of the finite element mesh.

The resulting coarse mesh reduced order model is as follows:

$$
A_{i} U_{i}=F_{i}+F_{D}\left(U_{i-1}\right)
$$

where now $i$ represents not only a configuration counter, but also a non-linear iteration or time step counter, and the correction for the model is evaluated in the previous non-linear iteration or time step, denoted by $i-1$. This approach assumes that close to non-linear convergence or when the time step is small enough, then:

$$
F_{D}\left(U_{i}\right) \approx F_{D}\left(U_{i-1}\right),
$$

and then the approximation is appropriate. For the neural network model, classical neural networks with sigmoid functions have been adopted in this work, although other possibilities may be explored. The used implementation is the one in the open source library FANN (see [26]).

\section{$3 \quad$ Numerical examples}

In this section some numerical examples are presented which illustrate the behavior of the proposed methodology. The first example deals with a large strain, dynamic, solid mechanics problem. Secondly, the method is applied to a nearly incompressible finite element flow problem. Finally, both reduced order models are put together in a fluid-structure interaction setting.

\subsection{Large strain, dynamic, solid mechanics problem}
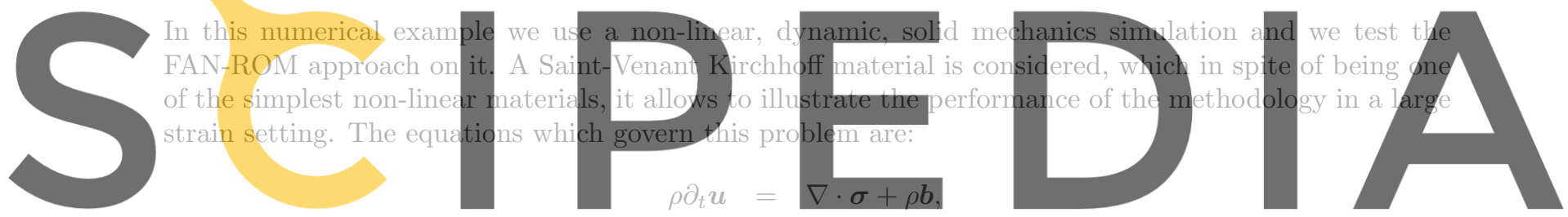

$\rho^{J}=\rho_{0}$,

Register for free at https//www.scipedia.com to download the version without the watermark

$$
F=\frac{\partial x}{\partial X}, \quad J=\operatorname{det}(F)
$$

where $\rho$ is the solid density ( $\rho_{0}$ the initial solid density), $\boldsymbol{u}$ is the velocity field, $\boldsymbol{\sigma}$ is the Cauchy stress tensor, $\boldsymbol{b}$ is the vector of external forces, $\boldsymbol{F}$ is the deformation gradient tensor, and $J$ its determinant. The constitutive equation for the Saint-Venant Kirchoff material, which relates displacements and stresses is as follows:

$$
\boldsymbol{S}=\lambda \operatorname{tr}(\boldsymbol{E}) \boldsymbol{I}+2 \mu \boldsymbol{E},
$$

where $\boldsymbol{S}$ is the second Piola-Kirchhoff stress tensor, which is related to the Cauchy stress tensor through the following expression:

$$
\boldsymbol{S}=J \boldsymbol{F}^{-1} \cdot \boldsymbol{\sigma} \cdot F^{-T},
$$

and $\boldsymbol{E}$ is the Green-Lagrange deformation tensor. For the solution of this problem, a large strain finite element formulation together with a Newton-Raphson linearization scheme is used.

The geometry of the considered beam and the load applied to it are depicted in Figure 1. The material parameters are taken as Young modulus $E=1000$, and Poisson ratio $\nu=0.21$.

The first test that we perform on this case is the training of a coarse mesh finite element model by using a static case. For this we solve 25 static simulation steps in which the vertical load is progressively increased from 0 to a value of 2.5. The solution for each of these steps is going to be used in order to train a coarse mesh model by using the FAN-ROM approach. Figures 2 and 3 show the comparison of the solution for the static case between the coarse mesh (78 elements) and a refined mesh (in this case two levels of uniform refinement are used, resulting in 1248 elements). Clearly, the coarse mesh is too dissipative which results in total displacements much smaller in the coarse mesh than in the fine one. 


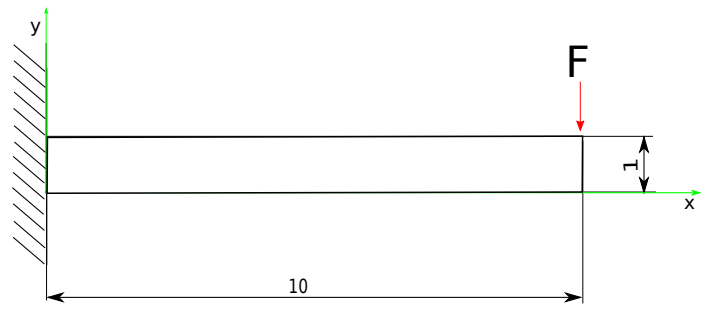

Figure 1: Geometry and load for the dynamic, large-strains beam example.

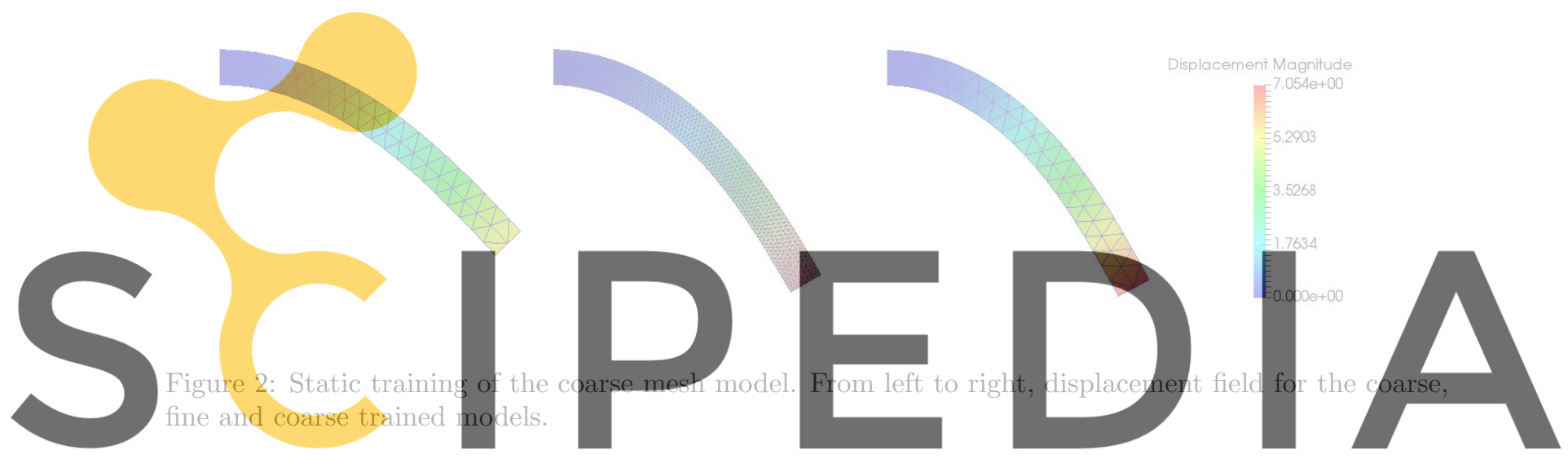

Register for free at https//www.scipedia.com to download the version without the watermark

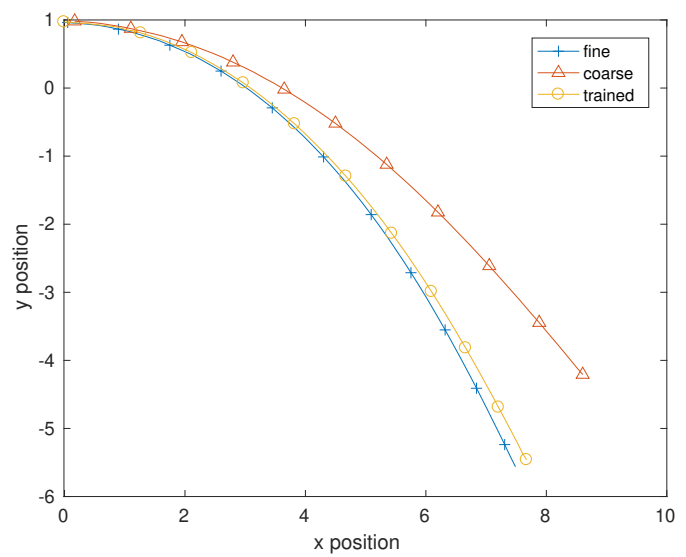

Figure 3: Displacement field in the upper surface of the beam for the coarse, fine and trained models. 

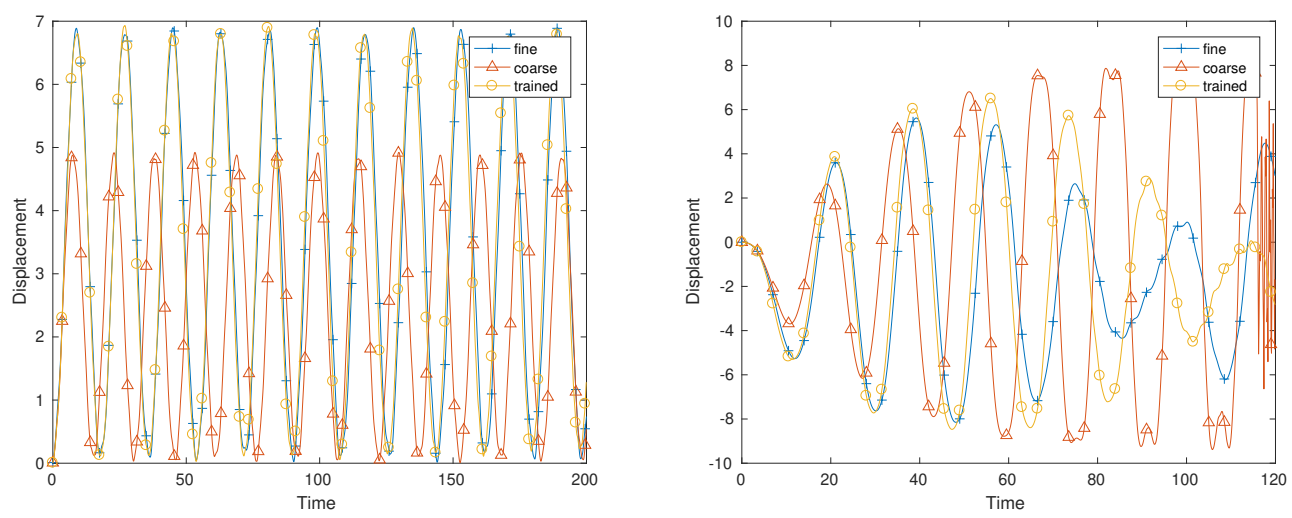

Figure 4: Application to a dynamic case. Left: static load. Right: Close to resonant dynamic load.

However, the same coarse mesh with the reduced order correction performs much more similarly to the fine mesh, and dissipation practically disappears, as shown in Figure 3.

The next test that we perform is to use the coarse mesh statically-trained model in a dynamic setting and to evaluate its performance. For this, a constant vertical load of modulus 1 is applied, and we compare the tip displacement of the coarse mesh model, the coarse mesh model statically trained with the FANROM methodology and the fine mesh model (Figure 4, left). The coarse model shows an important error both in amplitude and frequency. The FAN-ROM model, on the contrary, performs very similarly to the fine model, with almost no error in the oscillation frequency, and only a slight error in the oscillation amplitude. Regarding dor the fine mesh model is us emphasize again that tr used in this dvnamic configuration.

In the next example, one. The period of the
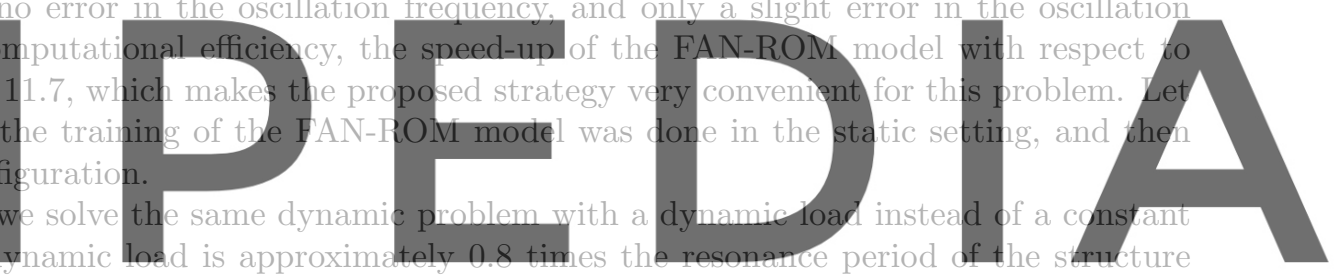

according to the fine mesh results. Figure 4, right, shows the comparison of the fine, coarse and (statically

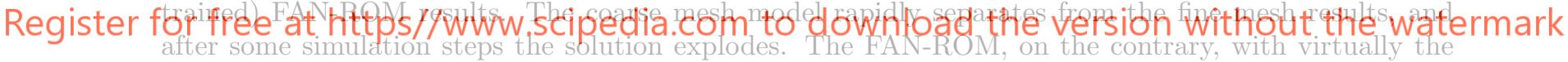
same computational cost, is capable of capturing the general behavior of the structure, although a phase difference accumulates through time integration.

\section{$3.2 \quad$ Fluid mechanics problem}

In this numerical example we solve a flow problem in a close to incompressible regime. The governing equations are:

$$
\begin{aligned}
\rho \partial_{t} \boldsymbol{u}+\rho \boldsymbol{u} \cdot \nabla \boldsymbol{u}-\mu \Delta \boldsymbol{u}+\nabla p & =\rho \boldsymbol{f} \\
\nabla \cdot \boldsymbol{u}+\epsilon \partial_{t} p & =0
\end{aligned}
$$

where $\rho$ is the fluid density, $\mu$ the viscosity, $p$ the pressure, $f$ the vector of body forces and $\epsilon$ is an artificial compressibility parameter. The incompressible limit corresponds to $\epsilon=0$. As it is well known, the incompressible flow limit gives rise to a saddle point problem (in the stationary and linear case). Although in our group we have extensively worked on stabilized formulations for this type of flows (see for instance the review in [16]), in this work the proposed reduced order model turns out to behave in an unstable manner when used together with a purely incompressible formulation, even if stabilization is used. This is the reason why in this numerical example a simpler problem where small values of $\epsilon$ are used is solved, what is known as an artificial compressibility approach. For the solution of this problem, the stabilized finite element formulation presented in $[14,15]$ is used.

In this numerical example we study the flow around a cylinder at $\mathrm{Re}=100$. The computational domain consists of a $16 \times 8$ rectangle with a unit-diameter cylinder centered at $(4,4)$. The horizontal inflow velocity is set to 1 at $x=0$. Slip boundary conditions which allow the flow to move in the direction 


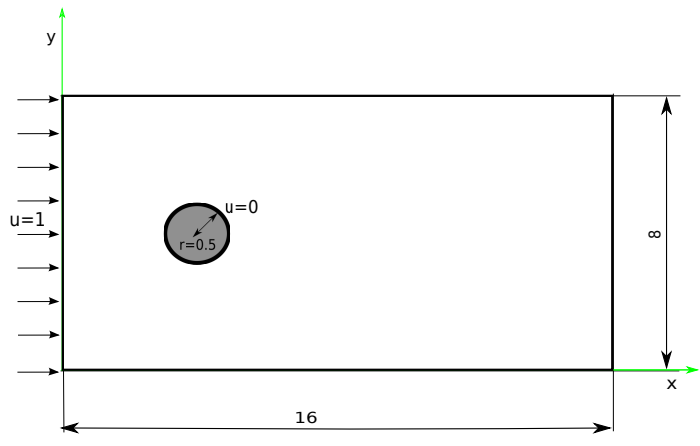

Figure 5: Flow around the cylinder geometry and boundary conditions.

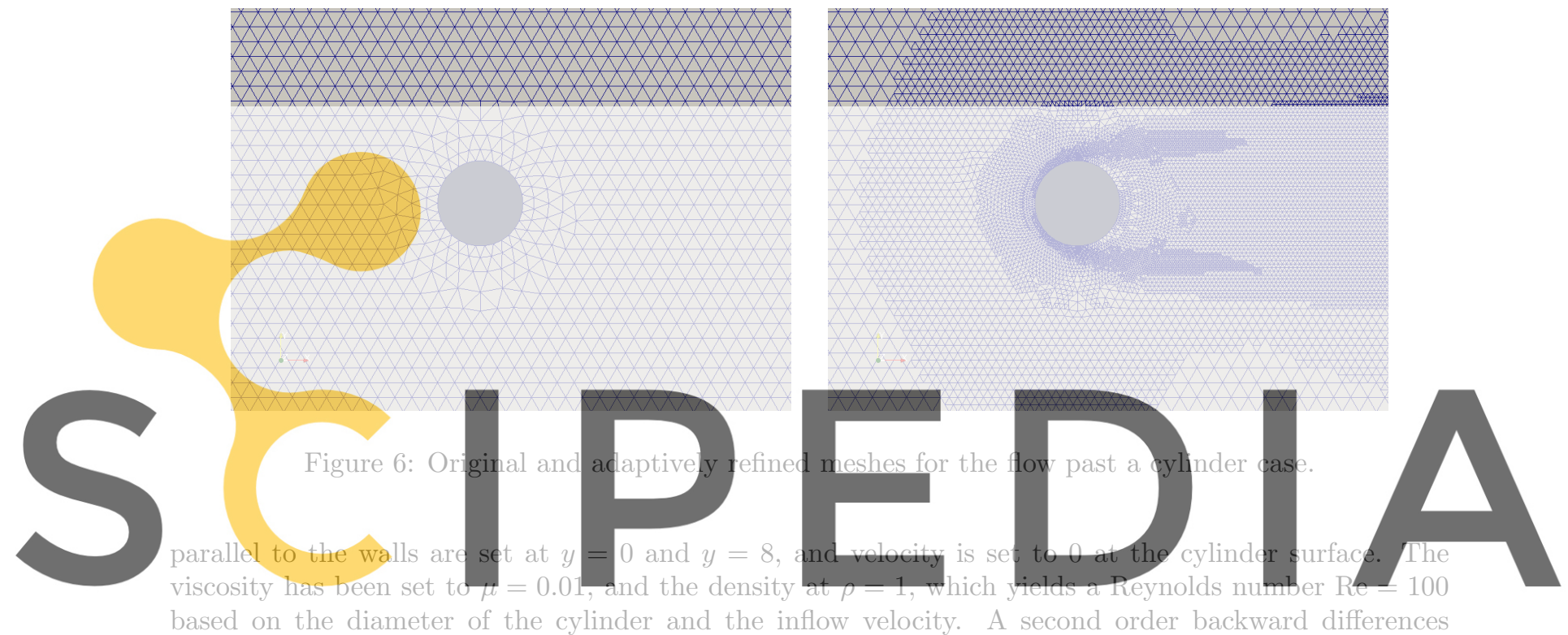

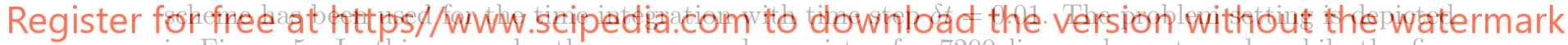

in Figure 5. In this example, the coarse mesh consists of a 7200 linear element mesh, while the fine

mesh has approximately 33000 adaptively refined elements. A comparison of the coarse and fine meshes

is depicted in Figure 6. As in the previous example, the information obtained by using the adaptively refined mesh is used as an input to train the FAN-ROM model on the coarse mesh. Figure 7 shows the instantaneous velocity and pressure fields; note that the problem is transient and non-stationary.

Figure 8 shows a comparison of the vertical velocity component and the pressure value at a point in the wake of the cylinder, for the coarse, adaptively refined, and coarse trained meshes. While the coarse mesh significantly departs from the fine model result, specially regarding the oscillation frequency and the absolute value of the pressure, the coarse trained model is able to perfectly recover the values obtained with the adaptively refined model.

Figure 9 shows a comparison of the drag and lift time history, for the coarse, adaptively refined, and coarse trained meshes. Again, the trained model is much more accurate than the coarse model without any training, although in this case the correction still yields some error in the drag computation. Regarding computational efficiency, the speed-up of the FAN-ROM model with respect to the fine mesh model is of 9.74 .

Despite the nice results obtained in this simple $R e=100$ example, we must emphasize that when applied to the same problem with $\mathrm{Re}=10,000$, we were not able to obtain reasonable results for the trained coarse model. Our guess is that this is possibly due to the impossibility of the coarse mesh of representing the fine mesh solution with a minimum of accuracy, and it poses a limitation for the proposed methodology, which needs to be addressed in the future. 

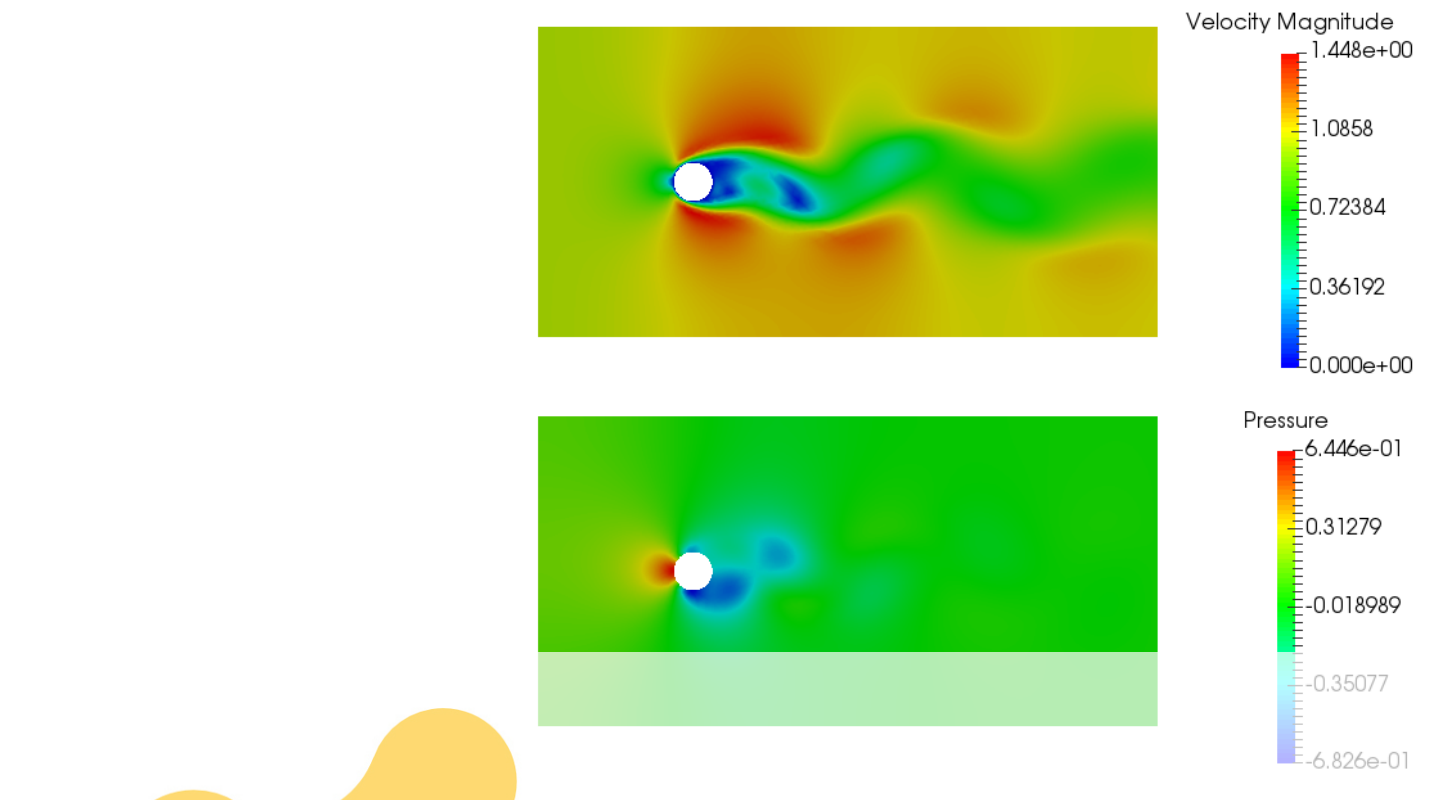

Figure 7: Velocity and pressure fields for the solution of the flow past a cylinder case.
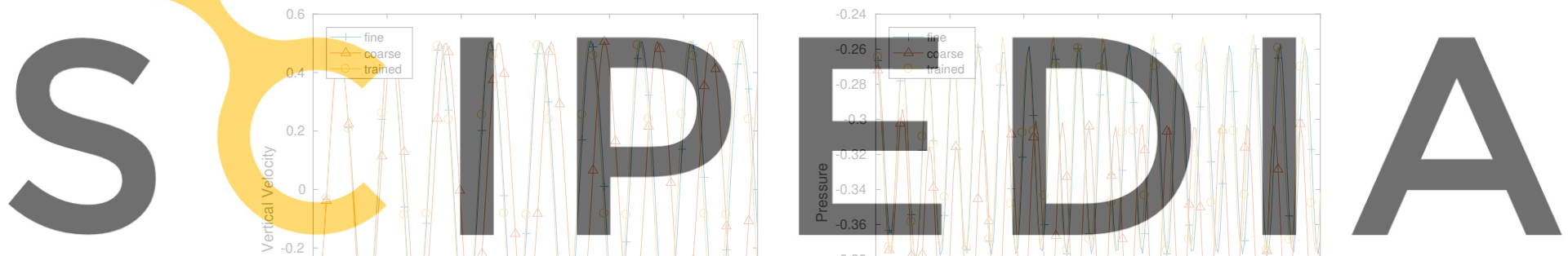

Register for free at https//www.scipedia.com to download the version without the watermark FAN-ROM models.
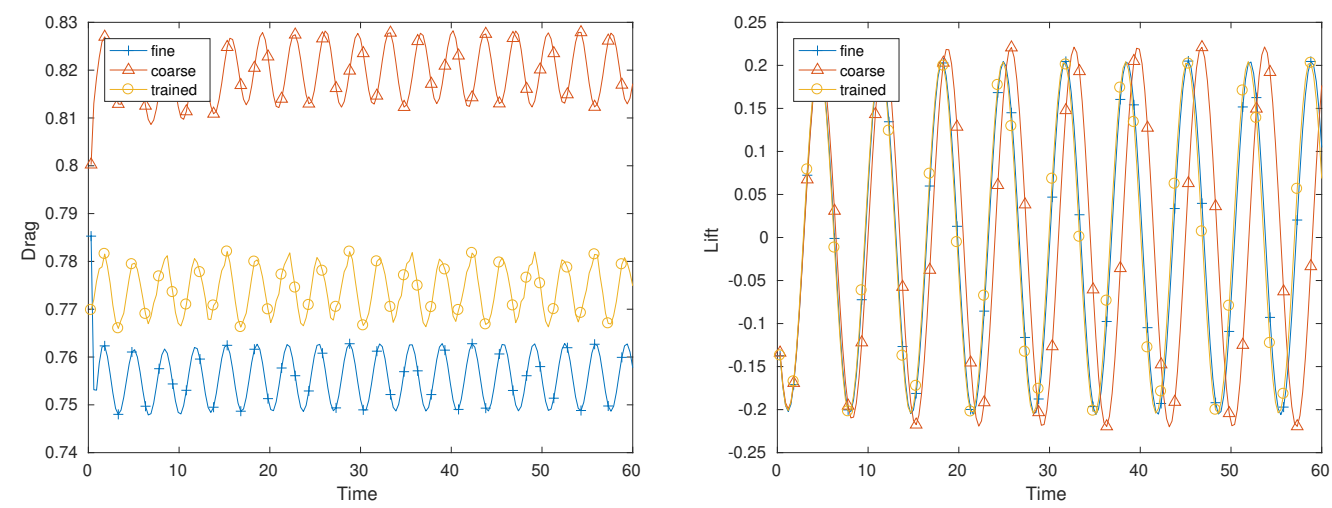

Figure 9: Comparison drag and lift time histories for the fine, coarse and FAN-ROM models. 


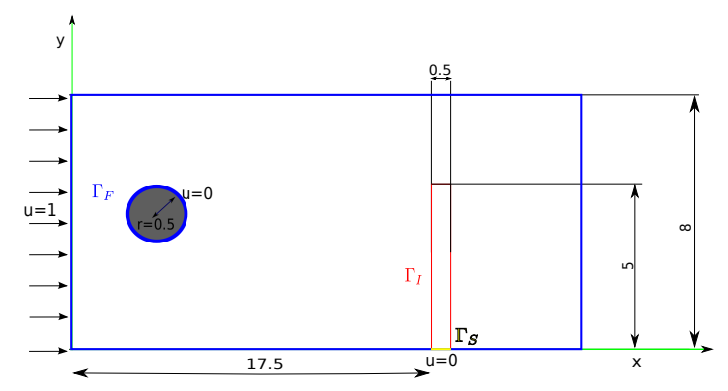

Figure 10: Geometry and boundary conditions for the FSI problem.

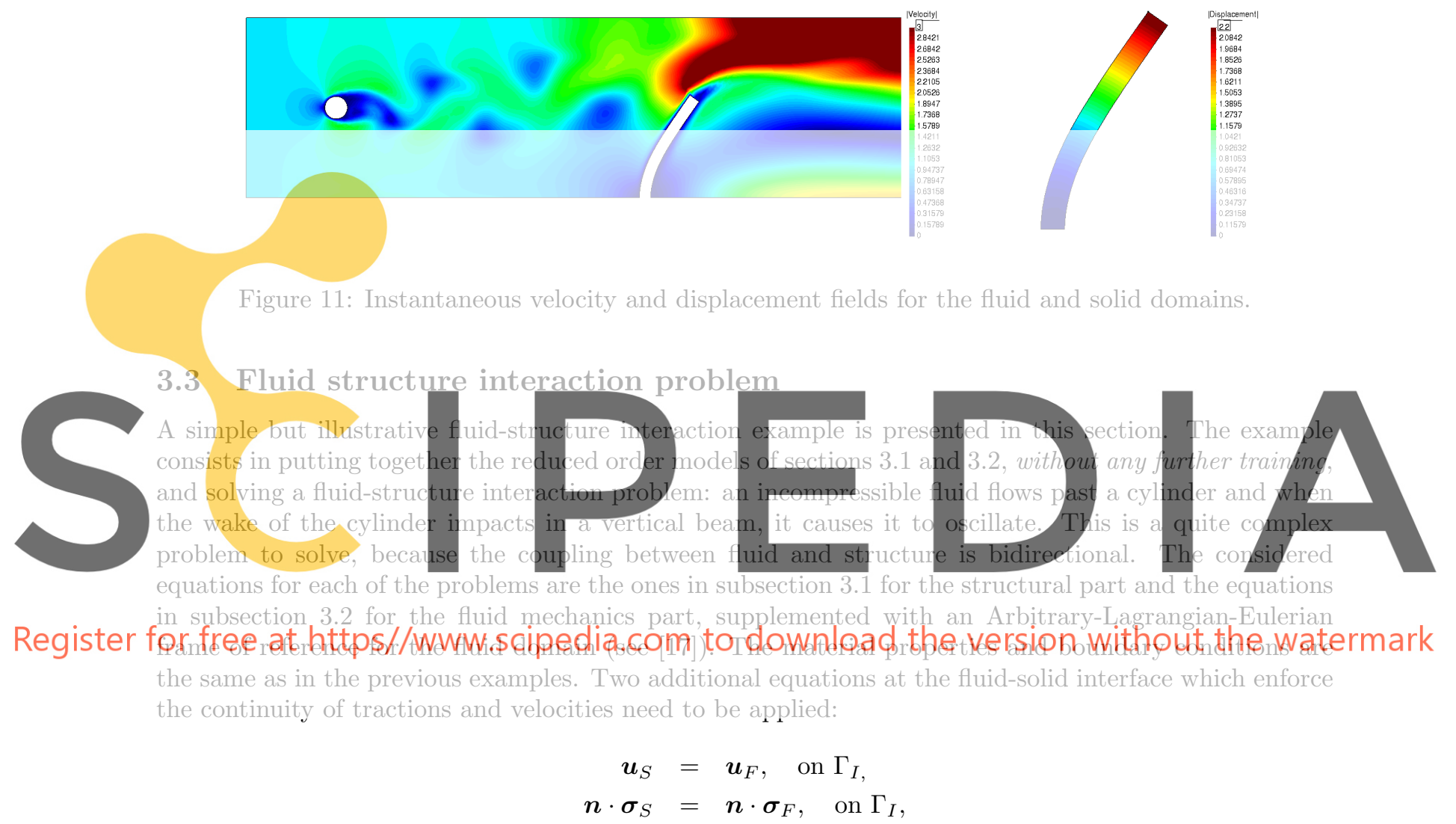

where $\boldsymbol{n}$ is the external normal to each domain, and the $S$ and $F$ subindexes represent solutions in the solid and fluid domain respectively, $\Gamma_{I}$ being the interface surface between both subdomains. In our partitioned coupling approach, the first condition is enforced strongly on the fluid domain at each iteration, while the second condition is enforced weakly on the solid domain, this is classically known as a Dirichlet-Neumann approach. Several iterations are required at each time step in order to reach the converged solution, in each of the iterations the position of each of the domains is updated. The solution of each problem is done by using a finite element formulation, in the case of the fluid mechanics problem stabilization is added to the Galerkin finite element method as explained in subsection 3.2.

The geometry and boundary conditions for this problem are shown in Figure 10. Figure 11 shows the instantaneous velocity and displacement profiles in the fluid and solid domains. The comparison between the coarse, fine and FAN-ROM results is shown in Figure 12. The time history of the displacement at the upper tip of the vertical beam is plotted for each model, after the dynamic stationary state is reached. The coarse model is quite diffusive, and underestimates the amplitude and mean value of the displacements. The FAN-ROM model, on the contrary, is capable of better reproducing the dynamic behavior, although it slightly overestimates the amplitude and frequency of the oscillations.

The conclusion of this numerical example is that the FAN-ROM is capable of adapting to configurations different from those for which it was trained. 


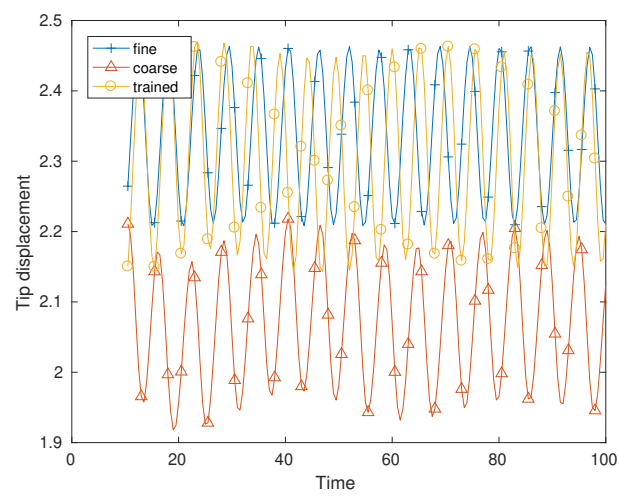

Figure 12: Comparison of the time history of the beam tip displacement for the coarse, fine and FAN-ROM reduced order model.

\section{Conclusions}

In this work a reduced order model based on adaptive finite element meshes and a correction term obtained by using an artificial neural network (FAN-ROM) has been presented. The idea is to run a high-fidelity simulation by using an adaptively refined finite element mesh, and compare the results obtained with those of a coarse mesh finite element model. From this comparison, a correction forcing term can be computed for each training configuration. A model for the correction term is built by using an artificial neural network, and the final reduced order model is obtained by putting together the coarse mesh finite element model, plus the artificial neural network model for the correction forcing term.

The methodology has been applied firstly to a non-linear solid mechanics problem, where we have departed from the training in a static configuration and then compared the results in a non-trained dynamic setting. The results in this case have been accurate, and an enhancement of the behavior of the reduced order model has been obtained, while maintaining a significant speed-up with respect to the high fidelity model.

The second problem to which the methodology has been applied is a transient quasi-incompressible flow. Due to the saddle point nature of the pure incompressible flow problem and its associated stability issues, a small penalty term has been added to the incompressibility constraint. This term has been necessary for the stability of the reduced order model. A simple flow past a cylinder problem has been tested, and good results have been obtained for $\mathrm{Re}=100$, but unstable behavior was observed when trying to apply the reduced order approach to a more complex Re $=10,000$ setting.

Finally, the two previous reduced order models have been put together in a fluid-structure interaction problem at $\operatorname{Re}=100$, without any further training. Good results have been obtained in this problem, showing the applicability of the artificial neural network trained reduced order model in configurations which are different from the training regime.

The results of the numerical examples show that the FAN-ROM is capable of improving the simulation results obtained in coarse finite element meshes at a reduced computational cost. The model is capable of adapting to configurations which differ from the training set, and as a consequence it can be useful in design processes in engineering, where iterative optimization is usually employed. However, the proposed FAN-ROM model has failed to reproduce fluid flow problems when the Reynolds number is high. The stability of the model in such settings and other complex problems will be a matter of future work.

\section{Acknowledgements}

J. Baiges gratefully acknowledges the support of the Spanish Government through the Ramón y Cajal grant RYC-2015-17367. R. Codina gratefully acknowledges the support received through the ICREA Acadèmia Research Program of the Catalan Government. E. Castillo acknowledges the support funded by the Chilean Council for Scientific and Technological Research through the proyect CONICYT-FONDECYT 11160160 . 


\section{References}

[1] I. Akhtar, J. Borggaard, and A. Hay. Shape sensitivity analysis in flow models using a FiniteDifference approach. Mathematical Problems in Engineering, 2010:1-23, 2010.

[2] S. Badia and J. Baiges. Adaptive finite element simulation of incompressible flows by hybrid continuous-discontinuous galerkin formulations. SIAM Journal on Scientific Computing, 35(1):A491A516, 2013.

[3] J. Baiges and C. Bayona. Refficientlib: An Efficient Load-Rebalanced Adaptive Mesh Refinement Algorithm for High-Performance Computational Physics Meshes. SIAM Journal on Scientific Computing, 39(2):65-95, 2017.

[4] J. Baiges, R. Codina, and S. Idelsohn. A Domain Decomposition strategy for Reduced Order Models. Application to the incompressible Navier-Stokes equations. Computer Methods in Applied Mechanics and Engineering, 267:23-42, 2013.

[5] J. Baiges, R. Codina, and S. Idelsohn. Explicit Reduced Order Models for the stabilized finite element approximation of the incompressible Navier-Stokes equations. International Journal for Numerical Methods in Engineering, 72:1219-1243, 2013.

[6] J. Baiges, R. Codina, and S. Idelsohn. Reduced-order subscales for POD models. Computer Methods in Applied Mechanics and Engineering, 291:173-196, 2015.

[7] J. Baiges, R. Codina, and S. Idelsohn. Reduced-order subscales for pod models. Computer Methods in Applied Mechanics and Engineering, 291:173-196, 2015.

[8] J. Baiges, R. Codina, A. Pont, and E. Castillo. An adaptive fixed-mesh ale method for free surface flows. Computer Methods in Applied Mechanics and Engineering, 313:159-188, 2017.

[9] M. Bergmann, L. Cordier, and J. Brancher. Drag minimization of the cylinder wake by trust-region proper orthogonal decomposition. Active Flow Control, 95:309-324, 2007.

[10] J. Burkardt, M. Gunzburger, and H. Lee. POD and CVT-based reduced-order modeling of NavierStokes flows. Computer Methods in Applied Mechanics and Engineering, 196(1-3):337-355, 2006.

[11] P. Chen and C. Schwab. Adaptive sparse grid model order reduction for fast bayesian estimation and inversion. In J. Garcke and D. Pflüger, editors, Sparse Grids and Applications - Stuttgart 2014, pages 1-27, Cham, 2016. Springer International Publishing.

[12] F. Chinesta, A. Ammar, and E. Cueto. Recent advances and new challenges in the use of the proper generalized decomposition for solving multidimensional models. Archives of Computational Methods in Engineering, 17:327-350, 2010.

[13] F. Chinesta, P. Ladeveze, and E. Cueto. A short review on model order reduction based on proper generalized decomposition. Archives of Computational Methods in Engineering, 18:395-404, 2011.

[14] R. Codina. A stabilized finite element method for generalized stationary incompressible flows. Computer Methods in Applied Mechanics and Engineering, 190:2681-2706, 2001.

[15] R. Codina. Stabilized finite element approximation of transient incompressible flows using orthogonal subscales. Computer Methods in Applied Mechanics and Engineering, 191:4295-4321, 2002.

[16] R. Codina, S. Badia, J. Baiges, and J. Principe. Variational multiscale methods in computational fluid dynamics. Encyclopedia of Computational Mechanics Second Edition, pages 1-28, 2018.

[17] J. Donea, A. Huerta, J. Ponthot, and A. Rodriguez-Ferran. Encyclopedia of computational mechanics vol. 1: Fundamentals., chapter 14: Arbitrary lagrangian-eulerian methods, 2004.

[18] Q. Du, V. Faber, and M. Gunzburger. Centroidal voronoi tessellations: Applications and algorithms. SIAM Review, 41:637-676, 1999.

[19] B. Hans-Joachim and M. Griebel. Sparse grids. Acta Numerica, 13:147-269, 2004. 
[20] G.-B. Huang, Q.-Y. Zhu, and C.-K. Siew. Extreme learning machine: Theory and applications. Neurocomputing, 70(1):489 - 501, 2006. Neural Networks.

[21] G. Kerschen, J.-c. Golinval, A. F. VAKAKIS, and L. A. BERGMAN. The method of proper orthogonal decomposition for dynamical characterization and order reduction of mechanical systems: An overview. Nonlinear Dynamics, 41(1):147-169, Aug 2005.

[22] J. Kou and W. Zhang. Multi-kernel neural networks for nonlinear unsteady aerodynamic reducedorder modeling. Aerospace Science and Technology, 67:309 - 326, 2017.

[23] F. Lihong. Review of model order reduction methods for numerical simulation of nonlinear circuits. Applied Mathematics and Computation, 167(1):576-591, 2005.

[24] D. J. Lucia, P. S. Beran, and W. A. Silva. Reduced-order modeling: new approaches for computational physics. Progress in Aerospace Sciences, 40(1):51 - 117, 2004.

[25] M. Mohebujjaman, L. Rebholz, and T. Iliescu. Physically constrained data-driven correction for reduced-order modeling of fluid flows. International Journal for Numerical Methods in Fluids, $89(3): 103-122,2019$.

[26] S. Nissen et al. Implementation of a fast artificial neural network library (FANN). Report, Department of Computer Science University of Copenhagen (DIKU), 31:29, 2003.

[27] S. M. Rahman, S. E. Ahmed, and O. San. A dynamic closure modeling framework for model order reduction of geophysical flows. 2019. Submitted.

[28] R. Reyes, R. Codina, J. Baiges, and S. Idelsohn. Reduced order models for thermally coupled low mach flows. Advanced Modeling and Simulation in Engineering Sciences, 5:1-20, 2018.

[29] G. Rozza, T. Lassila, and A. Manzoni. Reduced basis approximation for shape optimization in thermal flows with a parametrized polynomial geometric map. In J. S. Hesthaven and E. M. Ranquist, editors, Spectral and High Order Methods for Partial Differential Equations, volume 76, pages 307315. Springer Berlin Heidelberg, Berlin, Heidelberg, 2011.

[30] D. Ryckelynck. A priori hyperreduction method: an adaptive approach. Journal of Computational Physics, 202(1):346 - 366, 2005.

[31] D. Ryckelynck. Hyper-reduction of mechanical models involving internal variables. International Journal for Numerical Methods in Engineering, 77(1):75-89, 2009.

[32] O. San and R. Maulik. Extreme learning machine for reduced order modeling of turbulent geophysical flows. Phys. Rev. E, 97:042322, Apr 2018.

[33] O. San and R. Maulik. Machine learning closures for model order reduction of thermal fluids. Applied Mathematical Modelling, 60:681 - 710, 2018.

[34] O. San and R. Maulik. Neural network closures for nonlinear model order reduction. Advances in Computational Mathematics, 44(6):1717-1750, Dec 2018.

[35] A. Tello, R. Codina, and J. Baiges. Fluid structure interaction by means of variational multiscale reduced order models. International Journal for Numerical Methods in Engineering, 2019. Submitted.

[36] Q. Wang, J. S. Hesthaven, and D. Ray. Non-intrusive reduced order modeling of unsteady flows using artificial neural networks with application to a combustion problem. Journal of Computational Physics, 2019.

[37] X. Xie, M. Mohebujjaman, L. Rebholz, and T. Iliescu. Data-driven filtered reduced order modeling of fluid flows. SIAM Journal on Scientific Computing, 40(3):B834-B857, 2018. 